\title{
Sequential IVIM-Parametric as a Potential Image Biomarker in Evaluating the Radiosensitivity of Human Nasopharyngeal Carcinoma Xenografts
}

\author{
Youping Xiao ( $\nabla$ xyp999@yeah.net) \\ Department of Radiology, Fujian Medical University Cancer Hospital \& Fujian Cancer Hospital \\ Yunbin Chen \\ Department of Radiology, Fujian Medical University Cancer Hospital \& Fujian Cancer Hospital \\ Jianji Pan \\ Department of Radiation Oncology, Fujian Medical University Cancer Hospital \& Fujian Cancer Hospital \\ Ying Chen \\ Department of Radiology, Fujian Medical University Cancer Hospital \& Fujian Cancer Hospital \\ Xiang Zheng \\ Department of Radiology, Fujian Medical University Cancer Hospital \& Fujian Cancer Hospital \\ Dechun Zheng \\ Department of Radiology, Fujian Medical University Cancer Hospital \& Fujian Cancer Hospital \\ Qiaojuan Guo \\ Department of Radiation Oncology, Fujian Medical University Cancer Hospital \& Fujian Cancer Hospital \\ Yiqi Yao \\ Department of Radiology, Fujian Medical University Cancer Hospital \& Fujian Cancer Hospital
}

\section{Research Article}

Keywords: Intravoxel incoherent motion, Diffusion weighted imaging, Pathological features, Nasopharyngeal carcinoma, Xenograft model.

Posted Date: November 30th, 2021

DOI: https://doi.org/10.21203/rs.3.rs-1050632/v1

License: @ (i) This work is licensed under a Creative Commons Attribution 4.0 International License. Read Full License 


\begin{abstract}
BACKGROUND AND PURPOSE

Intravoxel incoherent motion (IVIM) is a considerable functional MR sequence in evaluated the tumor's response to chemo-radiotherapy. Our aim was to investigate the sequential IVIM parametric in assessing the dynamic changes of histopathological features on nasopharyngeal carcinoma (NPC) xenografts after receiving the fractional radiations.
\end{abstract}

\title{
EXPERIMENTAL APPROACH:
}

Sixty BALB/C-nu nude mice were transplanted with NPC cell lines of CNE-1 and CNE-2 to raise xenografts which further received the fractional radiations. On 3.0T MR system, IVIM (14 b-factors: $\left.0 \sim 1000 \mathrm{~s} / \mathrm{mm}^{2}\right)$ was performed on xegnorafts after radiations. IVIM-parametric of $D, f$ and $D *$ accompanied with the cellularity and necrosis proportion of NPC xenografts were calculated and analyzed respectively.

\section{KEY RESULTS}

NPC xenografts exhibited a larger $D$ and necrosis proportion coupled with a smaller $D^{*}, f$ and cellularity after fractional radiations, and CNE-2 xenografts presented greater changes than CNE-1 xenografts (all $P<0.01)$. Parametric $D$ and $f$ correlated negatively $(r=-0.824, P<0.001)$ while $D *$ correlated positively with $f(r=0.758, P<0.001)$ and $D(r=0.625, P=0.042)$. Moreover, $D$ correlated negatively with cellularity $\left(r_{s}=-0.861\right.$, $P<0.001)$ and positively with necrosis proportion $\left(r_{s}=0.952, P<0.001\right)$, $f$ behaved a positive correlation with cellularity $\left(r_{s}=0.627, P<0.001\right)$ and negative with necrosis proportion $\left(r_{s}=-0.649, P<0.001\right)$.

\section{CONCLUSIONS AND IMPLICATIONS:}

Sequential parametric derived from IVIM are valuable in characterizing the dynamic changes in histopathological features of NPC xenografts and can be utilized as a potential image biomarker for non-invasive assessment of tumor's radiosensitivity.

\section{Introduction}

Nasopharyngeal carcinoma (NPC) is a highly radio-sensitive head and neck malignancy that is endemic predominantly in Southeast Asia and Southern part of China. The efficacy and prognosis of NPC treated with radiations are closely related to tumor's intrinsic radiosensitivity which is greatly dependent on the tumorous microstructures and microenvironment ${ }^{[1,2]}$. NPC would exhibit a distinct tendency towards radioresistance when owning a highly invasive phenotype and histopathological features ${ }^{[2-4]}$. However, there still lack an effective and noninvasive imaging modality to assess the dynamic changes of histopathology as well as radiosensitivity of NPC underwent radiation therapy.

Indirectly, diffusion-weighted imaging (DWI) can characterize the micro-structures (i.e. cellularity and necrosis) by measuring the thermal motion of water molecular with the semi-quantitative parametric of apparent diffusion coefficient (ADC). However, ADC derived from the mono-exponential model of DWI can only reflect the combined features of water diffusion and perfusion effects in the capillary networks ${ }^{[5]}$. Even that the incoherent motion of spins can be described as the spatial "mixing" of spins in each image voxel, diffusion and perfusion are physically different phenomena in biological tissues ${ }^{[6]}$. Basing on the bi-exponential algorithm model, whereas, intravoxel incoherent motion (IVIM) allows the extraction of both water diffusion (i.e. D, pure diffusion coefficient) and perfusion of microcirculation (i.e. D*, perfusionrelated diffusion coefficient; and $f$, fraction of perfusion-related diffusion) simultaneously and separately $[5,6]$. In the past decade, there were increasing attractions on the diffusion and perfusion parametric of IVIM for characterizing the head and neck malignant tumors and predicting their response to relative treatments ${ }^{[7-11]}$. Recent evidences also suggested IVIM could provide an excellent ability to characterize tumor's microstructures and monitor their response to chemo-radiotherapy ${ }^{[10-13]}$. In a previous study on rodent model of glioma, $D$ and $f$ metrics correlated significantly with tumor's cellularity and vessel density at histology ${ }^{[14]}$. Nevertheless, there was only a very limited amount of study investigating the usefulness of IVIM-parametric in elucidating the pathological features of NPC xenografts as well as its intrinsic radiosensitivity.

In this present study, we intend to explore the application value of sequential IVIM-parametric in the assessment of dynamic changes of histopathological features in human NPC xenografts receiving the fractional radiations.

\section{Materials And Methods Ethics statement}


The experimental procedures conducted on the nude mice in this study was conducted in compliance with the institutional guidelines for the care and use of laboratory animals in Fuzhou General Hospital of Nanjing Military Command, Fuzhou, China, and approved by a grant from the licensing committee/institutional review board of Fujian Medical University Cancer Hospital. All mouse procedures were performed in accordance with the general policies determined by the National Institutes of Health Guide for Care and Use of Laboratory Animals. Moreover, the reporting in this manuscript follows the recommendations in the ARRIVE guidelines (PLoS Bio 8(6), e1000412, 2010).

\section{Human NPC xenograft models}

Sixty 4-week-old male Balb/C nude mice with immunological deficiency (Silaike Experimental Animal Co. Ltd., Shanghai, China) were used in this present study. All nude mice were maintained in a sterile environment, with $50 \%$ relative humidity and temperature at $25 \circ \mathrm{C}$, and divided randomly, blindly and equally into two independent groups, which were then transplanted with the human NPC cell lines of CNE-1 (a highly differentiated cell line, poor radiosensitivity) and CNE-2 (a poorly differentiated cell line, high radiosensitivity) to raise xenografts, respectively.

The CNE-1 and CNE-2 cell lines were provided by the Radiobiological Laboratory of Fujian Medical University Cancer Hospital and cultured in RPMI- 1640 medium supplemented with $100 \mathrm{U} / \mathrm{ml}$ penicillin, $100 \mu \mathrm{g} / \mathrm{ml}$ streptomycin, and $15 \%$ calf serum. Then, concentration of $1 \times 10^{7}$ cells were inoculated into the right armpit of nude mice to raise xenografts. The maximum diameters of xenografts were monitored and measured regularly after inoculation until they reached $1.5 \pm 0.2 \mathrm{~cm}$ before receiving the fractional radiations.

\section{Fractional radiations on NPC xenografts}

NPC xenografts received the fractional radiations by using the X-rays on the radiotherapy apparatus (Elekta-Compact linac, Shanghai, P.R. China). The protocols of fractional radiations were listed as follows: Firstly, the nude mice with xenografts were fixed in a prone position after the anesthetization by intraperitoneal injection of $2.5 \%$ chloral hydrate at a dose of $7.5 \mathrm{ul} / \mathrm{g}$ of body weight. According to the diameters of xenografts, different sizes of intraoral electron cones $(1 \sim 2 \mathrm{~cm})$ were selected and applied to protect the normal tissues beyond xenografts from radiations. The medical alginate dressing was applied to cover the surface of xenografts and the body of nude mice (except head) for equivalent compensation of radiations. Radiations were conducted on xenografts by using the linear accelerator of $6 \mathrm{MV} \mathrm{X-ray,} \mathrm{with} \mathrm{the}$ source-skin distance (SSD) was $100 \mathrm{~cm}$ and the field size of irradiation was of $3 \mathrm{~cm} \times 3 \mathrm{~cm}$, and the single dose of irradiation was 10Gy with a dose rate of $350 \mathrm{mGy} / \mathrm{min}$. The whole course of irradiation was supervised under the closed-circuit television surveillance in case the animal under light anesthesia moved out or escaped from the irradiation field. PTW-UNIDOS-T10002 type dosimeter coupled with type ionization chamber (PTW-31014) were performed for the measurement of absorbed dose accordingly.

The xenografts of CNE-1 and CNE-2 were further sub-classified into the following five subgroups: (i) $G_{0}$ group, no radiation (control); (ii) $G_{1}$ group, 10 Gy radiation dose; (iii) $G_{2}$ group, 20 Gy radiation dose; (iv) $G_{3}$ group, 30 Gy radiation dose; (v) $G_{4}$ group, 3 days after 30 Gy radiation exposure; respectively. Each subgroups included six nude mice with human NPC xenografts. The prescription of fractional irradiation was of a single fraction of $10 \mathrm{~Gy}$ at the alternative days.

\section{MR imaging protocols and image analysis}

Anesthesia described above was performed again on nude mice with xenografts before the MR scanning. On a 3.0T MR system (Achieva TX, Philips Healthcare, Best, The Netherlands), a special 4-channel animal coil (Model: CG-MUC 18-H300-AP, Shanghai Chenguang Medical Technology Co., Shanghai, China) was employed in the MR scanning of xenografts. The axial IVIM-DWI sequence coupled with the short T1 inversion recovery-T2 weighted imaging (T2WI-STIR) of axial and coronal planes were conducted on $\mathrm{G}_{0}$ xenografts directly, $\mathrm{G}_{1} \sim \mathrm{G}_{3}$ xenografts in next day after radiations and $\mathrm{G}_{4}$ xenografts in the third day after radiations. The relevant scanning parameters were listed as following: i) T2WI-STIR sequence, with a Echo time (TE)/Repetition time (TR) of $60 \mathrm{~ms} / 6898 \mathrm{~ms}$, Flip angle $90^{\circ}$, a Field of view (FOV) of $40 \times 40 \mathrm{~mm}^{2}$, a acquisition matrix of $240 \times 240$, and a Number of Signal Average (NSA) of 2; ii) Coronal T2-weighted TSE sequence, with a TR/TE of $1500 / 180 \mathrm{~ms}$, a slice thickness of $4 \mathrm{~mm}$, an inter-slice gap of $0 \mathrm{~mm}$, a FOV of $80 \times 40 \mathrm{~mm}^{2}$, an acquisition matrix of $240 \times 240$ and a NEX of 2; iii) IVIM-DWI sequence, 14 b-factors $\left(0,10,20,30,40,50,100,150,200,350,500,650,800\right.$, and $\left.1000 \mathrm{~s} / \mathrm{mm}^{2}\right)$, Free-breathing; Single-shot, Three diffusion directions $(x, y, z)$, with a TR/TE of $3000 \mathrm{~ms} /$ shortest, a FOV of $40 \times 40 \mathrm{~mm}^{2}$, a NSA of 4 , a Flip angle of $90^{\circ}$, a Matrix of $108 \times 156$, a slice thickness $5 \mathrm{~mm}$, an inter-slice gap of $1 \mathrm{~mm}$, and a total 6 slices. The total acquisition time consumed 13 minutes 30 seconds. As aforementioned, $G_{0}$ group received MRI scan without any irradiation. $G_{1}, G_{2}$, and $G_{3}$ groups received MRI scan on the next day after irradiated with 10,20 and 30 Gy doses, respectively. $G_{4}$ groups underwent MRI scans on the third day, respectively, after receiving a total dose of 30 Gy.

The images of IVIM-DWI acquired were saved as PAR format and then transmitted to the extension workstation (EWS, Philips Healthcare, Best, The Netherlands). The IVIM-parametric (i.e. pure diffusion coefficient (D), perfusion related diffusion coefficient ( $\left.D^{\star}\right)$ and fraction of perfusion related diffusion (f)) were analyzed and calculated with the software of Interactive Data Language(IDL) 6.3 (Research Systems Inc.; Boulder, CO, USA), using a bi-exponential model with the equation ${ }^{[5]}: S_{b} / S_{0}=(1-f) \times \exp (-b D)+f \times \exp \left(-D^{*}\right)$. Two experienced radiologists 
engaged in interpreting MR images and measuring the IVIM-parametric with a double-blind control. The regions of interest (ROIs) were firstly drawn manually on the maximum cross-sectional area of target lesions on the morphology images of T2WI-STIR and then copied automatically to the parametric maps of $D, f$ and $D^{*}$ to derived the relevant parametric (See Fig. 1), respectively. The ROI should be contoured along the margin of xenografts so far as possible including the whole tumor, and care should be taken to avoid the intralesional necrosis as well as the surrounding structures (i.e. bone and air). The volume of xenografts were measured automatically on the axial T2WI-STIR images at the EWS workstation. The $D$ of $G_{x}$ xenografts was defined as $D_{x}(x=0 \sim 4)$. The definitions of $D^{*}$ and $f$ parametric were established in the same way of $D$.

\section{Histopathological examinations}

All nude mice were euthanized by cervical dislocation after their relative MR scans according to the American Veterinary Medical Association (AVMA) Guidelines for the Euthanasia of Animals (2020). Then, NPC xenografts were harvested from necropsy and tissue samples were formalin-fixed and paraffin-embedded for histopathological examinations. The macroscopic specimens of xenografts were then cut into 4$\mu \mathrm{m}$ microscopic slices for the pathological and immunohistochemical analysis. Three consecutive microscopic slides in each groups were randomly chosen and stained with hematoxylin-eosin (H-E) with the standard pathological procedure described in [15]. The RGB pathological images were captured on an optical microscope with magnification factors of 40,200 and 400, respectively, and then saved as the JPEG files of $2448 \times 1920 \times 24$ bit. On each slides, five complete and non-overlapping fields of views with magnification factors of 40 and 400 were randomly selected for in-depth microstructural analysis, and then transformed into black-and-white images to calculated the cellularity or necrosis proportion of xenografts $(\times 100 \%)$ automatically by using the image $\mathrm{J}$ software.

\section{Statistical analysis}

Statistical analyses was conducted on the SPSS18.0 software (IBM Corporation, Armonk, New York, USA). The Kolmogorov-Smirnov (K-S) test was applied to analysis the normality distributions of quantitative data. Intraclass correlation coefficient (ICC) was calculated to evaluate the data variability between two observers. One-Way ANOVA compared the difference of IVIM-parametric and pathological features between multiple groups, and Student's $t$ test or Mann-Whitney $U$ test compared the difference between two independent groups. The correlation between variables was tested with Pearson correlation or Spearman rank correlation analysis. $\mathrm{P}$ value $<0.05$ was considered statistically significant.

\section{Results}

\section{Tumor volumes and IVIM-parametric of NPC xenografts}

The R2 fit goodness (g) on the measurements of IVIM-parametric ranged from 0.976 to 0.983 . The inter- and intra-observer ICCs of $D, D^{*}$ and f parametric calculated on NPC xenografts were of $0.973 \mathrm{vs} .0 .988,0.851 \mathrm{vs}$. 0.908 , and $0.855 \mathrm{vs} .0 .938$, respectively; and their corresponding coefficient variations (CVs) were of $9.26 \%$ vs. $8.69 \%, 24.12 \%$ vs. $15.95 \%$, and $23.71 \%$ vs. $12.78 \%$, respectively (See Fig. 2).

Table 1 summarized the tumor volumes and IVIM-parametric in different groups of CNE-1 and CNE-2 xenografts. The tumor volume of $\mathrm{G}_{0}$ between CNE-1 and CNE-2 xenografts were consistent $(P=0.303)$, whereas, $C N E-2$ xenografts exhibited a greater changes on tumor volumes after fractional radiations $(P<0.001)$, but not for $C N E-1$ xenografts $(P=0.198)$. Moreover, tumor volumes in $G_{3}$ and $G_{4}$ of $C N E-2$ xenografts were significantly smaller than those of CNE-1 xenografts ( $P=0.048$ and 0.021 , respectively). 
Table 1

The pre- and post-radiation IVIM-parametrics and tumor volumes in the subgroups of NPC xenografts with different radiosensitivity

\begin{tabular}{|c|c|c|c|c|c|c|c|}
\hline $\begin{array}{l}\text { IVIM- } \\
\text { parametrics }\end{array}$ & Xenografts & $\mathbf{G}_{0}$ & $\mathbf{G}_{1}$ & $\mathrm{G}_{2}$ & $\mathrm{G}_{3}$ & $\mathrm{G}_{4}$ & $P$ \\
\hline \multirow{2}{*}{$\begin{array}{l}\text { Tumor } \\
\text { volume } \\
\left(\mathrm{mm}^{3}\right)\end{array}$} & CNE-2 & $328.538 \pm 122.091$ & $324.000 \pm 75.860$ & $272.214 \pm 52.432$ & $238.556 \pm 37.397$ & $215.286 \pm 48.131$ & $<0.001$ \\
\hline & CNE-1 & $309.167 \pm 66.793$ & $304.143 \pm 63.813$ & $295.286 \pm 62.174$ & $275.600 \pm 26.576$ & $270.750 \pm 31.521$ & 0.198 \\
\hline \multirow{2}{*}{$\begin{array}{l}\text { D } \\
\left(\times 10^{-3}\right. \\
\left.\mathrm{mm}^{2} / \mathrm{s}\right)\end{array}$} & CNE-2 & $1.091 \pm 0.193$ & $0.979 \pm 0.323$ & $1.141 \pm 0.354$ & $1.263 \pm 0.323$ & $1.453 \pm 0.426$ & 0.008 \\
\hline & CNE-1 & $1.014 \pm 0.254$ & $0.994 \pm 0.185$ & $1.042 \pm 0.214$ & $1.080 \pm 0.241$ & $1.155 \pm 0.310$ & 0.28 \\
\hline \multirow{2}{*}{$\begin{array}{l}f \\
(\%)\end{array}$} & CNE-2 & $0.219 \pm 0.065$ & $0.213 \pm 0.069$ & $0.185 \pm 0.073$ & $0.154 \pm 0.023$ & $0.141 \pm 0.011$ & 0.001 \\
\hline & CNE-1 & $0.202 \pm 0.058$ & $0.198 \pm 0.088$ & $0.186 \pm 0.072$ & $0.186 \pm 0.046$ & $0.185 \pm 0.090$ & 0.666 \\
\hline \multirow{2}{*}{$\begin{array}{l}D^{*} \\
\left(\times 10^{-3}\right. \\
\left.\mathrm{mm}^{2} / \mathrm{s}\right)\end{array}$} & CNE-2 & $112.371 \pm 27.412$ & $110.656 \pm 33.258$ & $97.246 \pm 37.701$ & $81.161 \pm 21.504$ & $68.573 \pm 17.730$ & $<0.001$ \\
\hline & CNE-1 & $109.810 \pm 28.486$ & $106.326 \pm 49.849$ & $96.089 \pm 29.835$ & $95.170 \pm 34.534$ & $90.768 \pm 22.597$ & 0.34 \\
\hline $\begin{array}{l}\mathrm{G}_{0} \sim \mathrm{G}_{4} \text { repres } \\
\text { IVIM=Intravo }\end{array}$ & the non-rac & $\begin{array}{l}\text { ation group, and grc } \\
\text { otion; NPC=Nasoph } \\
\text { ent. }\end{array}$ & $\begin{array}{l}\text { of } 10 \mathrm{~Gy}, 20 \mathrm{~Gy}, 3 \\
\text { yngeal carcinoma }\end{array}$ & $\begin{array}{l}D \text { Gy radiation and } \\
D=\text { Pure diffusion }\end{array}$ & $\begin{array}{l}\text { days after } 30 G y \text { ra } \\
\text { efficient; } f=\text { Fractior }\end{array}$ & diation, respectivel & \\
\hline
\end{tabular}

For IVIM-parametric, parametric D of CNE-1 xenografts appeared a slight increase after fractional radiations and the general changes were not significant $(\mathrm{P}=0.28)$. On the contrary, $C N E-2$ xenografts presented a brief decline on parametric $\mathrm{D}$ after $10 \mathrm{~Gy}$ radiation following with a obvious increase after $20 \mathrm{~Gy}$ radiation, the general changes were significant $(P=0.008)$. Parametric $D_{3}$ and $D_{4}$ of $C N E-2$ xenografts were significantly higher than those of CNE-1 xenografts $(P=0.012 ; 0.002)$. Moreover, parametric $f$ and $D *$ of $C N E-1$ xenografts declined slightly after radiations but the general changes were not significant $(P=0.666 ; 0.34)$, whereas, $f$ and $D *$ of $C N E-2$ xenografts declined significantly after 20Gy irradiation $(P=0.001 ;<0.001)$, and $f_{4}$ coupled with $D^{*}{ }_{4}$ of $C N E-2$ xenografts were significantly lower than $C N E-1$ xenografts (P=0.001; 0.003) (Table 1 and Fig. 3).

\section{The pathological and hypoxia-related features in NPC xenografts}

The general distributions of cellularity and necrosis proportion were detailed in Table 2.

Table 2

The pre- and post-radiation pathological features in the subgroups of NPC xenografts with different radiosensitivity

\begin{tabular}{|c|c|c|c|c|c|c|}
\hline \multirow[t]{2}{*}{ Groups } & \multicolumn{3}{|l|}{ Cell density(\%) } & \multicolumn{3}{|c|}{ Necrosis proportion(\%) } \\
\hline & CNE-1 & CNE-2 & $P$ value & CNE-1 & CNE-2 & $P$ value \\
\hline $\mathbf{G}_{0}$ & $0.852 \pm 0.023$ & $0.805 \pm 0.024$ & 0.056 & $0.307 \pm 0.028$ & $0.284 \pm 0.096$ & 0.068 \\
\hline $\mathrm{G}_{1}$ & $0.864 \pm 0.022$ & $0.823 \pm 0.023$ & 0.081 & $0.300 \pm 0.014$ & $0.309 \pm 0.033$ & 0.436 \\
\hline $\mathrm{G}_{2}$ & $0.857 \pm 0.021$ & $0.749 \pm 0.049$ & 0.004 & $0.328 \pm 0.054$ & $0.465 \pm 0.088$ & 0.131 \\
\hline $\mathrm{G}_{3}$ & $0.846 \pm 0.034$ & $0.728 \pm 0.068$ & 0.001 & $0.347 \pm 0.046$ & $0.532 \pm 0.127$ & $<0.001$ \\
\hline $\mathrm{G}_{4}$ & $0.810 \pm 0.055$ & $0.551 \pm 0.170$ & $<0.001$ & $0.357 \pm 0.043$ & $0.710 \pm 0.066$ & $<0.001$ \\
\hline$P$ value & 0.087 & $<0.001$ & & 0.094 & $<0.001$ & \\
\hline
\end{tabular}

The general changes of cellularity and necrosis proportion of CNE-2 xenografts after radiations were statistically significant ( $\mathrm{P}<0.001$ for both), but not for the $C N E-1$ xenografts $(P=0.087 ; 0.094)$ (Table 2 and Fig. 4). The cellularity and necrosis proportion of $\mathrm{G}_{0}$ between $\mathrm{CNE}^{-1}$ and CNE-2 xenografts were consistent $(P=0.056 ; 0.068)$. Whereas, the necrosis proportion of xenografts dramatically increased especially after $20 \mathrm{~Gy}$ radiation, and necrosis proportion of $\mathrm{G}_{3}$ and $\mathrm{G}_{4}$ xenografts of $\mathrm{CNE}-2$ xenografts were significantly higher than those of $\mathrm{CNE}-1$ 
xenografts ( $P<0.001$ for both). Similarly, the cellularity of xenografts declined obviously after $20 \mathrm{~Gy}$ radiation, and the cellularity of $G_{2}, G_{3}$, and $\mathrm{G}_{4}$ in $\mathrm{CNE}-1$ xenografts were much higher relative to $\mathrm{CNE}-2$ xenografts $(\mathrm{P}=0.004 ; 0.001 ;<0.001$; respectively) (See Table 2 , Fig. 4 and 5$)$.

\section{Correlations between IVIM-parametric and pathological features.}

The correlation analysis revealed that $D$ and $f$ parametric were negatively correlated $(r=-0.824, P<0.001)$, while parametric $D^{*}$ was positively correlated with $f(r=0.758, P<0.001)$ and $D(r=0.625, P=0.042)$. On the other hand, parametric $D$ and the cellularity correlated negatively $\left(r_{s}\right.$ $=-0.861, P<0.001)$, and the necrotic proportion was positively correlated with $D\left(r_{s}=0.952, P<0.001\right)$. Besides, $f$ was positively correlated with the cellularity $\left(r_{s}=0.627, P<0.001\right)$, but negatively with the necrosis proportion $\left(r_{s}=-0.649, P<0.001\right)$. Moreover, $D *$ presented a weak positive correlation with the cellularity $\left(r_{s}=0.280, P=0.011\right)$ and a weak negative correlation with the necrosis proportion $\left(r_{s}=-0.335, P=0.002\right)$.

\section{Discussion}

The present study sequentially analyzed the dynamic changes of IVIM-parametric coupled with the histopathological features in different radio-sensitive NPC xenografts underwent the fractional radiations. After radiations, CNE-2 xenografts of higher radiosensitivity presented greater changes on the IVIM-parametric than lower radiosensitive CNE-1 xenografts, as well as the histopathological features. Parametric D correlated negatively with cellularity and positively with necrotic proportion of xenografts, whereas, $f$ correlated positively with cellularity and negatively with necrosis proportion. It is suggested sequential IVIM-parametric could provide valuable bio-information in characterizing the histopathological features of NPC xenografts.

To evaluate the heterogeneity of diffusion in vivo effectively, Le Behan $D$ et al. ${ }^{[5,6]}$ proposed a theory of IVIM basing on a bi-exponential model. As for IVIM-parametric, D represents the true diffusion of water molecular in extracellular space (i.e. slow diffusion pool), D* characterizes the perfusion-related diffusion of microcirculation in the capillary networks (i.e. fast diffusion pool) and $f$ is the fraction of perfusion-related diffusion, respectively. Thus, the semi-quantitative IVIM-parametric could further clarify and distinguish the contribution of fast diffusion pool from that of slow diffusion pool basing on the bi-exponential model of IVIM theory ${ }^{[16,17]}$. In this study, we observed a transient decline on D parametric after the fractional radiation of 10Gy, which then increased continuously. However, the cellularity and necrosis proportion of xenografts didn't change significantly until 20Gy radiations. The potential reason might be that early in the course of irradiation, the irradiation-related cell swelling was of common phenomenon among tumor tissues. Before the process of necrocytosis or apoptosis, the expression of $\mathrm{Na}^{+}-\mathrm{K}^{+}$-ATPase on the membrane firstly decreased dramatically due to the hypoxia condition in tumor tissues. Even that the integrity of membrane was not affected, the trans-membrane transport of water molecule has declined markedly, leading to the swelling of tumor cells and a narrower extracellular space, and making the aggravated restriction of water diffusion in extra-cellular space. Pan $\mathrm{J}$ et al. ${ }^{[18]}$ had reported a obvious decline on ADC value of NPC xenografts early after a single fraction of radiations, which is consistent with our present results.

As the process of fractional radiation moving forward, the Caspase 3 would be activated to further motivate the process of cell apoptosis due to the accumulation of irradiation dose ${ }^{[19,20]}$. Therefore, the cellularity of tumor tissues declined while the necrosis areas increased much more obviously, and the freedom of water diffusion was less restricted, resulting in a notable increase on parametric $\mathrm{D}$. On the other hand, radiations cause the swelling of vascular endothelial cell in the capillary networks as well, resulting in a more serious microcirculation disturbance and down-regulating the perfusion level in tumor tissues. Accordingly, $D^{*}$ and $f$ of xenografts exhibited a significant decline after fractional radiations, in particular, the later stage of irradiation. In addition, the corresponding changes of parametric $D, f$, and $D^{*}$ after radiations were found more greater in CNE-2 xenografts of higher radiosensitivity relative to the lower radiosensitive CNE-1 xenografts. These above observations suggested dynamic changes of IVIM-parametric could indirectly characterize the microstructures and radiosensitivity, and potentially provide a bio-information on tumor tissues of NPC xenografts receiving the fractional radiations.

Previous studies have demonstrated IVIM-parametric not only useful for characterizing the diffusion and perfusion features of malignancies, but also correlated well with patient's treatment response to chemotherapy and/or radiotherapy ${ }^{[10-13]}$. Our preliminary results indicated IVIMparametric could effectively reflect the radiosensitivity of NPC xenografts and potentially clarify their dynamic changes of microstructures after fractional radiations. D correlated negatively with cellularity and positively with necrosis proportion, and $f$ exhibited a positive correlation with cellularity and a negative correlation with necrosis proportion. Similarly, Puig J et al. ${ }^{[21]}$ contended IVIM-parametric were useful for characterizing the diffusion and perfusion features of glioblastoma, in which they observed a significant positive correlation between $f$ and microvessel density as well as a negative correlation between cellularity and $D$. As confirmed by histology, parametric $f$ and $D *$ were associated siginificantly with the angiogenesis degree of microvessels and determined upon the attenuation of microvascular, in particular, the basement membrane thickness coupled with pericyte coverage. Thus, $D *$ parametric was accounted as a proportional 
correlation with the average blood velocity as well as capillary segment length ${ }^{[22-24]}$. These founding indirectly revealed that regions with the highest cellularity in tumor tissues always correlated with the maximum vascularity area, and maps of IVIM-parametric could also highlight tumor's heterogeneous pathological features generally.

Nevertheless, importantly, it is consistent with previous reports that a relatively higher coefficient variations (CVs) were observed on parametric $D^{*}$ and $f$, ranging from $12.78-24.12 \%$. Lai $V$ et al. ${ }^{[7,25]}$ also believed the higher variability in the measurement of parametric $D^{*}$ and f lay heavily on the method of drawing ROI manually. Therefore, in this present study, two radiologists conducted the analysis and calculation of IVIM-parametric with a double-blind control to reduce the variability and error of measurements. Besides, two observers initially reached an agreement on the edge definition of target lesions before manually contouring the ROI. It was suggested the ROI should be delineated along the edge so far as possible to include the whole tumor of xenografts, paying attentions to avoid the interference of the intralesion necrosis, adjacent bone, air, and other structures, etc. The inter- and intra-observer ICCs for parametric $D, D *$ and $f$ were observed ranging from 0.851 to 0.973 , indicating a excellent reproducibility and consistency on the measurements of IVIM-parametric between observers in this present study.

This study still has several limitations. Firstly, the IVIM-parametric and pathological features of xenografts were derived and originated from different irradiation groups, and there always existed a certain degree of heterogeneity between xenograft's tissues themselves. Another limitation is that the ROIs placed on images of IVIM-parametric were drawn manually including the whole tumor of xenografts, whereas, the pathological features analyzed on HE images were selected randomly which is partly representative and could not reflect the heterogeneity of integrated tumor tissues as the IVIM sections. Moreover, the observation period is not enough to characterize the xenograft's microenvironment since the dynamic changes of IVIM-parametric and pathological features might continue for such an extended period after radiations.

\section{Conclusion}

High-radiosensitive CNE-2 xenografts exhibited greater changes on IVIM-parametric and microstructures than poor-radiosensitive CNE-1 xenografts after receiving the fractional radiations. In particular, parametric $D$ and $f$ correlated significantly with the pathological features of xenografts. Thus, sequential IVIM-parametric can provide valuable information in characterizing the dynamic changes of histopathological features for NPC xenografts tissues after irradiation and might be utilized as a potential biomarker for the non-invasive assessment of NPC tumor's radiosensitivity.

\section{Declarations}

\section{Acknowledge}

This study was initially supported by the Joint Funds for the innovation of science and Technology, Fujian province (Grant No.2017Y9078, and (2020)729) and partly supported by Science and Technology Program of Fujian Province, China (Grant No.2018Y2003) and Fujian Provincial Clinical Research Center for Cancer Radiotherapy and Immunotherapy(Grant No.2020Y2012).

\section{The authors have no conflicts of interest to disclose.}

\section{References}

1. Hu Y, E H, Yu X, Li F, Zeng L, Lu Q, Xi X, Shen L. Correlation of quantitative parameters of magnetic resonance perfusion-weighted imaging with vascular endothelial growth factor, microvessel density and hypoxia-inducible factor-1ain nasopharyngeal carcinoma: Evaluation on radiosensitivity study[J]. Clin Otolaryngol. 2017; 00: 1-9. doi: 10.1111/coa.12982.

2. Chen W, Hu GH. Biomarkers for enhancing the radiosensitivity of nasopharyngeal carcinoma[J]. Cancer Biol Med. 2015; 12(1): $23-32$.

3. Zhang G, Wang W, Yao C, Zhang S, Liang L, Han M, Ren J, Qi X, Zhang X, Wang S, Li L. Radiation-resistant cancer stem-like cell properties are regulated by PTEN through the activity of nuclear $\beta$-catenin in nasopharyngeal carcinoma[J]. Oncotarget, 2017, 8(43): 74661-74672.

4. Zhao Y, Shen L, Huang X, Jing D, Huang D, Fu J, Li Z, Zhang G, Shen L. High expression of Ki-67 acts a poor prognosis indicator in locally advanced nasopharyngeal carcinoma[J]. Biochem Biophys Res Commun. 2017; 494(1-2):390-396.

5. Le Bihan D, Breton E, Lallemand D, Grenier P, Cabanis E, Laval-Jeantet M. MR imaging of intravoxel incoherent motions: application to diffusion and perfusion in neurologic disorders[J]. Radiology. 1986; 161 (2): 401-7.

6. Le Bihan D, Breton E, Lallemand D, Aubin ML, Vignaud J, Laval-Jeantet M.Separation of diffusion and perfusion in intravoxel incoherent motion MR imaging [J]. Radiology. 1988; 168(2): 497-505. 
7. Lai V, Li X, Lee VH, Lam KO, Fong DY, Huang B, Chan Q, Khong PL. Nasopharyngeal carcinoma: comparison of diffusion and perfusion characteristics between different tumour stages using intravoxel incoherent motion MR imaging [J]. Eur Radiol. 2014 Jan;24(1):176-183.

8. Ai QY, King AD, Chan JSM, Chen W, Chan KCA, Woo JKS, Zee BCY, Chan ATC, Poon DMC, Ma BBY, Hui EP, Ahuja AT, Vlantis AC, Yuan J. Distinguishing early-stage nasopharyngeal carcinoma from benign hyperplasia using intravoxel incoherent motion diffusion-weighted MRI [J]. Eur Radiol. 2019 Oct;29(10):5627-5634.

9. So TY, Ai QH, Lam WKJ, Qamar S, Poon DMC, Hui EP, Mo FKF, Chan KCA, King AD. Intravoxel incoherent motion diffusion-weighted imaging for discrimination of benign and malignant retropharyngeal nodes [J]. Neuroradiology. 2020 Dec;62(12):1667-1676.

10. Xiao Y, Pan J, Chen Y, Chen Y, He Z, Zheng X. Intravoxel Incoherent Motion-Magnetic Resonance Imaging as an Early Predictor of Treatment Response to Neoadjuvant Chemotherapy in Locoregionally Advanced Nasopharyngeal Carcinoma[J]. Medicine (Baltimore). 2015; 94(24): e973.

11. Paudyal R, Oh JH, Riaz N, Venigalla P, Li J, Hatzoglou V, Leeman J, Nunez DA, Lu Y, Deasy JO, Lee N, Shukla-Dave A. Intravoxel incoherent motion diffusion- weighted MRI during chemoradiation therapy to characterize and monitor treatment response in human papillomavirus head and neck squamous cell carcinoma[J]. J Magn Reson Imaging. 2017; 45(4) :1013-1023.

12. Xiao Y, Chen Y, Chen Y, He Z, Yao Y, Pan J. Longitudinal Assessment of Intravoxel Incoherent Motion Diffusion Weighted Imaging in Evaluating the Radio-sensitivity of Nasopharyngeal Carcinoma Treated with Intensity-Modulated Radiation Therapy [J]. Cancer Res Treat. 2019; 51(1):345-356.

13. Qamar S, King AD, Ai QH, So TY, Mo FKF, Chen W, Poon DMC, Tong M, Ma BB, Hui EP, Yeung DK, Wang YX, Yuan J. Pre-treatment intravoxel incoherent motion diffusion-weighted imaging predicts treatment outcome in nasopharyngeal carcinoma [J]. Eur $\mathrm{J}$ Radiol. 2020;129:109127.

14. lima M, ReynaudO, TsurugizawaT, Ciobanu L, Li JR, Geffroy F, Djemai B, Umehana M, Le Bihan D. Characterization of glioma microcirculation and tissue features using intravoxel incoherent motion magnetic resonance imaging in a rat brain model [J]. Invest Radiol. 2014; 49: 485-490.

15. Kmiec Z. J.A. Kiernan. Histological and Histochemical Methods: Theory and Practice. 5th edition, Scion Publishing, 2015,571 pp. Folia Histochem Cytobiol. 2016; 54(1) :58-59.

16. Le Bihan D. Apparent diffusion coefficient and beyond: what diffusion MR imaging can tell us about tissue structure[J]. Radiology.2013; 268(2): 318-22.

17. Le Bihan D. Diffusion, confusion and functional MRI [J]. Neuroimage. 2012; 62(2): 1131-1136.

18. Pan J, Zang L, Zhang Y, Hong J, Yao Y, Zou C, Zhang L, Chen Y. 49HEarly changes in apparent diffusion coefficients predict radiosensitivity of human nasopharyngeal carcinoma xenografts[J]. Laryngoscope. 2012; 122 (4): 839-43.

19. Wang F, Tang J, Li P, Si S, Yu H, Yang X, Tao J, Lv Q, Gu M, Yang H, Wang Z. Chloroquine Enhances the Radiosensitivity of Bladder Cancer Cells by Inhibiting Autophagy and Activating Apoptosis[J]. Cell Physiol Biochem. 2017;45(1):54-66.

20. Varghese R, Majumdar A, Kumar G, Shukla A. Rats exposed to $2.45 \mathrm{GHz}$ of non-ionizing radiation exhibit behavioral changes with increased brain expression of apoptotic caspase 3. Pathophysiology. 2017 Nov 14. pii: S0928-4680(17):30052-4.

21. Puig J, Sánchez-González J, Blasco G, Daunis-I-Estadella P, Federau C, Alberich-Bayarri Á, Biarnes C, Nael K, Essig M, Jain R, Wintermark M, Pedraza S. Intravoxel Incoherent Motion Metrics as Potential Biomarkers for Survival in Glioblastoma. PLoS One. 2016;11(7):e0158887.

22. Price SJ, Young AM, Scotton WJ, Ching J, Mohsen LA, Boonzaier NR, Lupson VC, Griffiths JR, McLean MA, Larkin TJ. Multimodal MRI can identify perfusion and metabolic changes in the invasive margin of glioblastomas. J Magn Reson Imaging. 2016 ; $43(2)$ : $487-494$.

23. Dominietto M, Lehmann S, Keist R, Rudin M. Pattern analysis accounts for heterogeneity observed in MRI studies of tumor angiogenesis. Magn Reson Med. 2013; 70(5):1481-1490.

24. Cui Y, Zhang C, Luo R, Liu H, Zhang Z, Xu T, Zhang Y, Wang D. Noninvasive monitoring of early antiangiogenic therapy response in human nasopharyngeal carcinoma xenograft model using MRI with RGD- conjugated ultrasmall superparamagnetic iron oxide nanoparticles [J]. Int J Nanomedicine. 2016; 11:5671-5682.

25. Lai V, Li X, Lee VH, Lam KO, Chan Q, Khong PL. Intravoxel incoherent motion MR imaging: comparison of diffusion and perfusion characteristics between nasopharyngeal carcinoma and post-chemoradiation Fibrosis [J]. Eur Radiol. 2013; 23(10): $2793-801$.

\section{Figures}



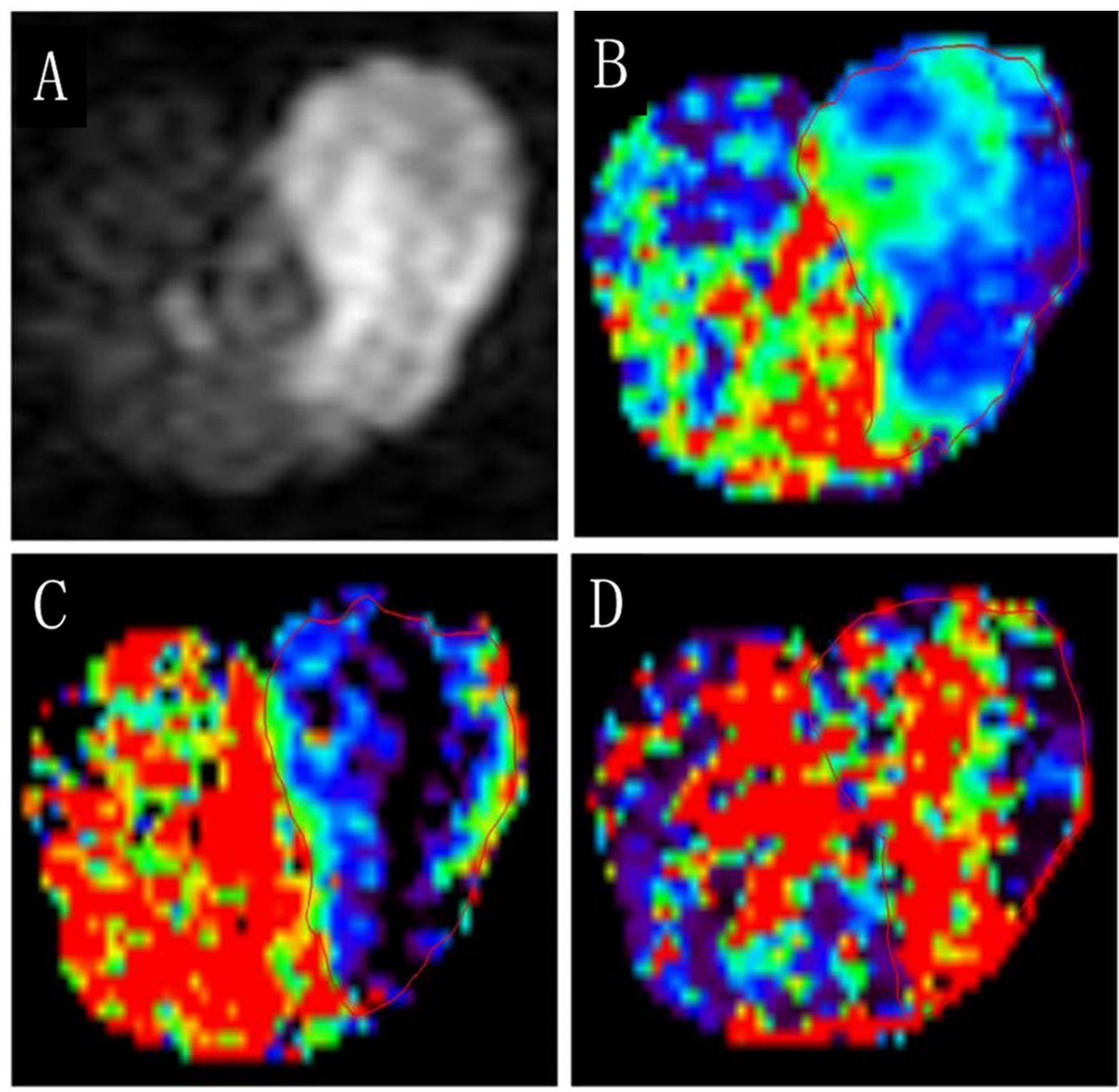

\section{Figure 1}

Map $A$ is the conventional IVIM image (b factor=800 s/mm2) whereas map B D are the IVIM-parametric images of $D, f$ and $D *$, respectively. The region of interest (ROI) (red curve) is firstly drawn manually along the border of NPC xenograft on the conventional IVIM image (high signal intensity) and then copied automatically to the images of $D, f$ and $D *$ to derived the relevant IVIM-parametric, respectively. 

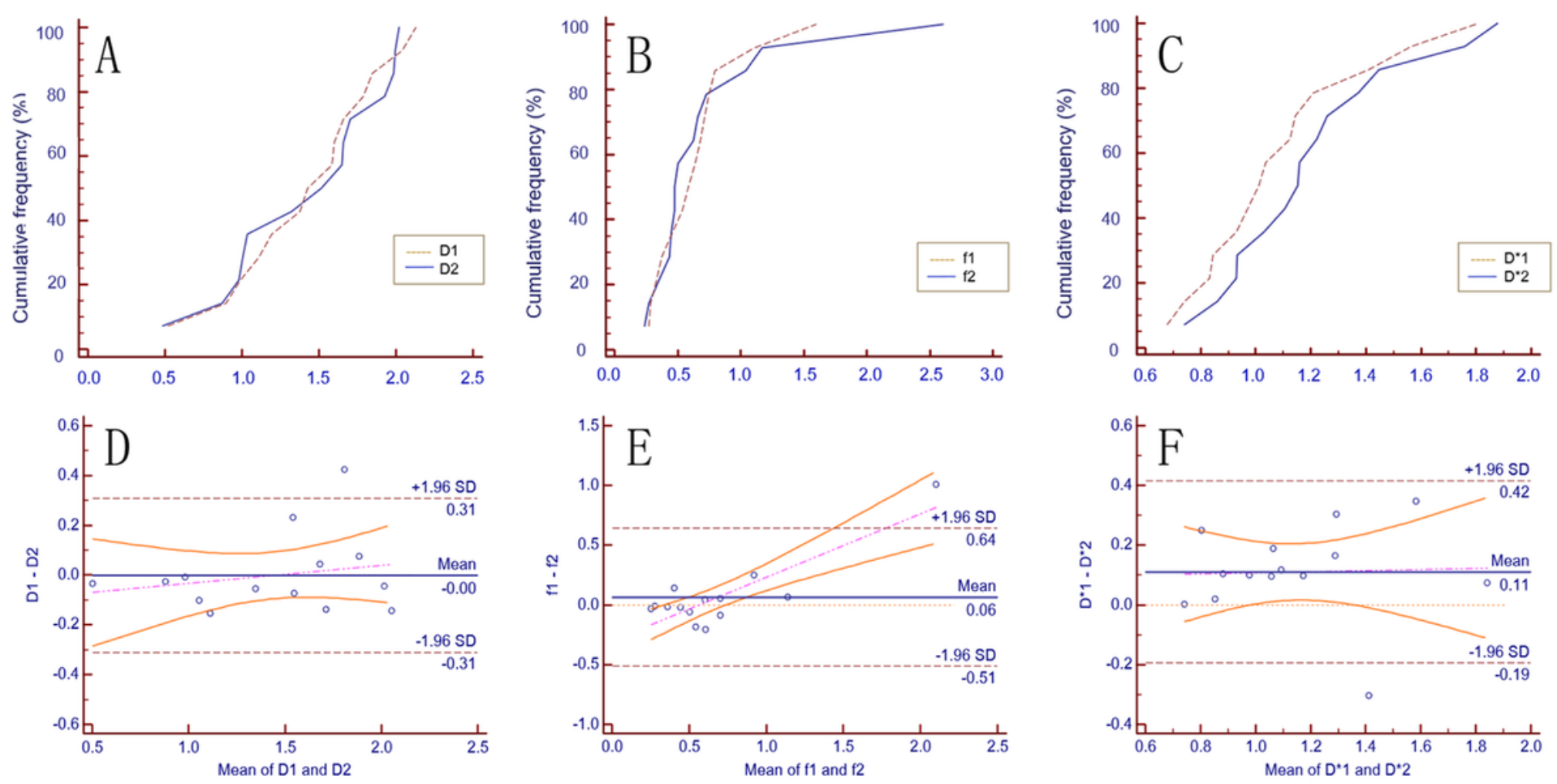

\section{Figure 2}

The curves represent the results of intraclass coherent coefficient (ICC) analysis of the measurements on IVIM-parametric of D (map A and $D$ ), $f\left(\right.$ map $B$ and $E$ ) and $D^{*}$ (map D and F), respectively. The results indicates that the IVIM-parametric are of excellent reproducibility on the measurements conducted by two independent observers.

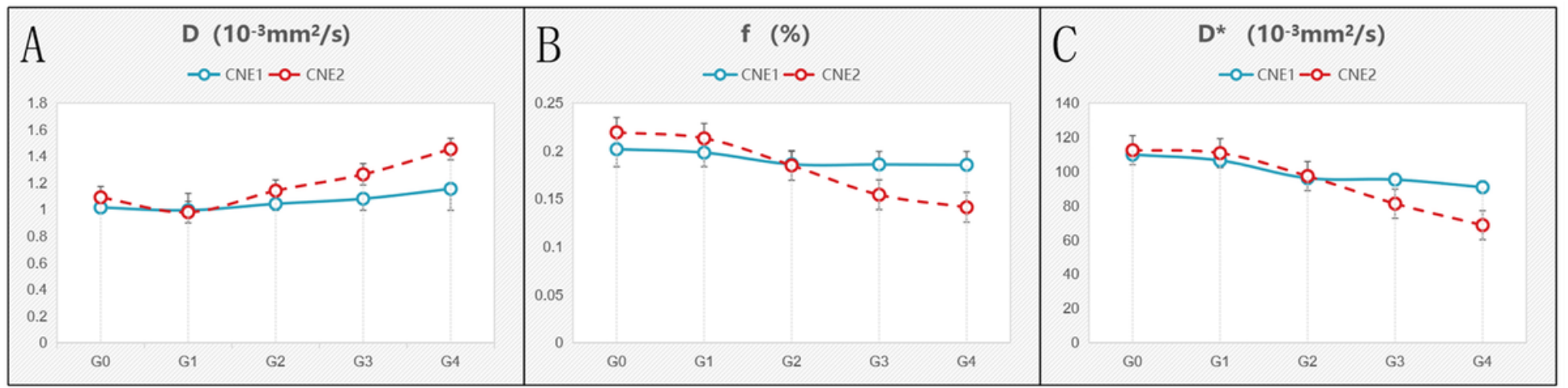

\section{Figure 3}

Map A C show the comparison of the curves of dynamic changing features on the IVIM-parametric D (map A), $f($ map B) and D* (map C) between CNE-1 xenografts (blue continuous curve) and CNE-2 xenografts (red dotted curve) throughout the course of fractional radiations, respectively. 


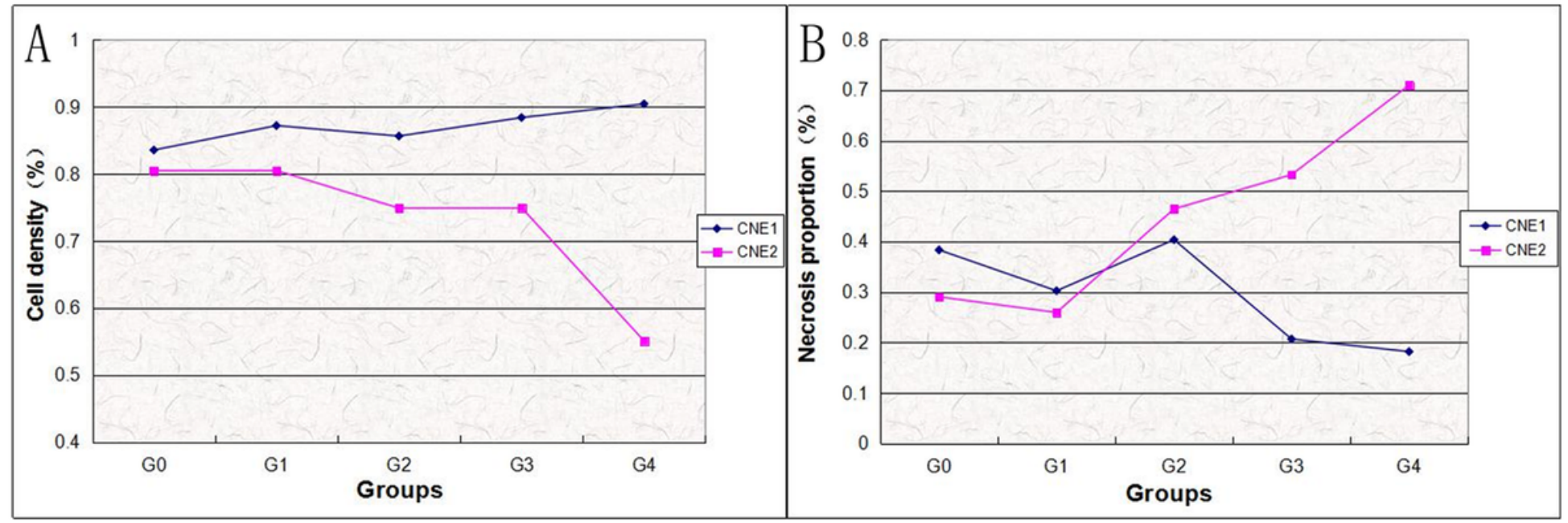

\section{Figure 4}

Map A and B show the comparative curves of dynamic changing features on the cellularity (map A) and necrosis proportion (map B) between CNE-1 xenograft (blue curve) and CNE-2 xenografts (red curve) after receiving the fractional radiations.

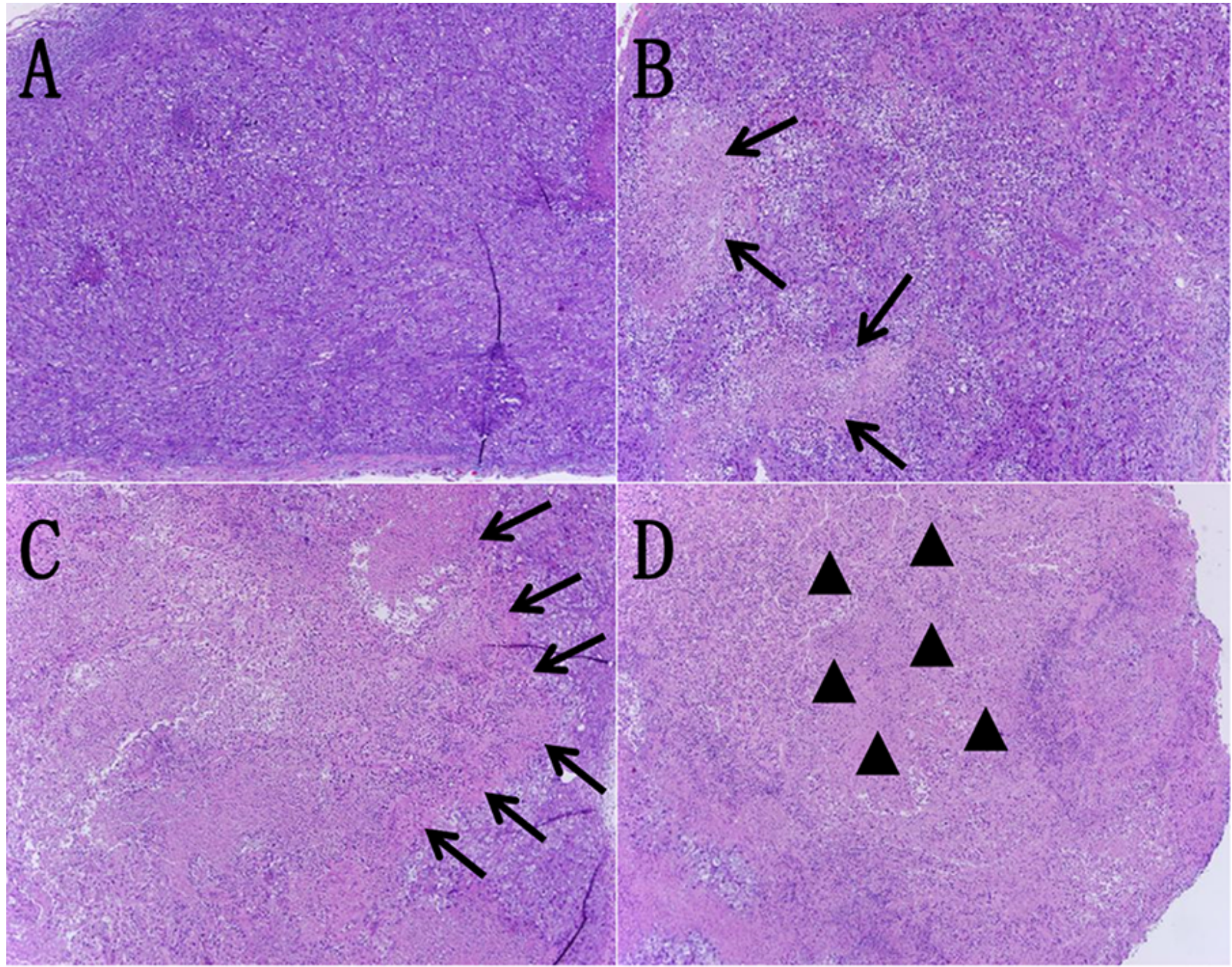

\section{Figure 5}

Map A D are the lower magnification views $(\mathrm{HE}, \times 40)$ of histopathological images (A囚no radiation; B: 10Gy radiation; C: 20Gy radiation; D: $30 \mathrm{~Gy}$ radiation; respectively), showing the dynamic changes on the necrosis proportion and cellularity of CNE- 2 xenografts after receiving the 
fractional radiations. The necrosis proportion of CNE-2 xenografts increased continuously (black arrows and triangles) and the cellularity of xenografts declined dramatically after irradiation. 